\title{
Mitigating Energy Hole based Routing Algorithm for Wireless Sensor Networks
}

\author{
${ }^{1}$ Sibi Amaran, ${ }^{2}$ Dr. R. Madhan Mohan, Dr.R.Jebakumar \\ ${ }^{1}$ Research Scholar, Department of Computer Science and Engineering, Annamalai University. \\ ${ }^{2}$ Associate Professor, Department of Computer Science and Engineering, Annamalai University. \\ ${ }^{3}$ Associate Professor, Department of CSE, SRM Institute of Science and Technology, \\ Kattankulathur. \\ sibi.amaran@gmail.com, madhanmohan_mithu@yahoo.com,jebakumr@srmist.edu.in
}

\begin{abstract}
In wireless sensor networks (WSN), the energy hole problem needs to be resolved to considerably improve the network lifetime. This paper presents an efficient Mitigating Energy Hole based routing algorithm (MEHRA) for WSN. Initially, the network initialization process takes place where the entire network is partitioned into few regions and is again divided into sectors with respect to equiangular wedges. During the partitioning of the network into regions and wedges, Head Node (HN) will be chosen for all sectors regarding distance and energy. Besides, the sensor nodes in a provided sector form a chain to communicate with their HN. The construction of chain process has begun from the farther node in the $\mathrm{HN}$ and all nodes will choose their chain neighboring by the execution of greedy algorithm. The HN collects the data in its predecessor and its individual sector and transmits the aggregated data to its successor $\mathrm{HN}$ till it reaches the BS. An extensive set of experimental analysis was carried out to highlight the supremacy of the MEHRA technique. The obtained simulation outcome ensured the goodness of the MEHRA technique interms of distinct aspects.
\end{abstract}

Keywords: Energy hole problem, WSN, Routing technique, Base station, Head node

\section{Introduction}

Wireless Sensor Networks (WSNs) comprise a group of resource limited sensor nodes [1], which are applied extensively in domains like ecological observation, armed forces, disaster prediction, farming, remote patient observation, factory mechanization, and so on. The primary role of the sensor nodes is to gather the related data from atmosphere and forward them to Base Station (BS) or sink by multi-hop transmission. The nodes placed nearby the sink in multi-hop WSNs should forward maximum traffic when compared with alternate distant nodes. The topological demerits result in huge power exhaustion and led to energy holes or hot spot problems. Consequently, external layer sensor nodes are not applicable to send the data to BS and it limits the network duration even under the presence of maximum Residual Energy (RE). Fig. 1 portrays the overview of energy hole problem where the nodes placed nearby BS are comprised of minimum residual battery power, and nodes placed away from the sink are composed of maximum residual battery power. Also, energy hole problem occurs when the nodes receive or transmit massive data packets when compared with alternate nodes [2]. In multi-hop system, the entire system is confused because of the energy hole problem. Node deployment is the basic step involved in developing sensor network. Actually, sensor nodes are battery powered and placed randomly in the defied region. One of the major challenges in sensor nodes is battery power constraints, computation of energy limitations, repeat data collection, and insufficient storage of the system. Optimizing power utilization is a crucial task in WSNs which helps in expanding the network duration. This problem can be addressed by many developers and concentrate on resolving the problem significantly. When the sensor nodes are placed evenly, nodes placed closer to the BS forward the data, and the data gathered alternate nodes are placed far away from BS, in multi-hop fashion. At this point, sensor nodes deployed nearby the BS applies maximum power and results in premature node death. 


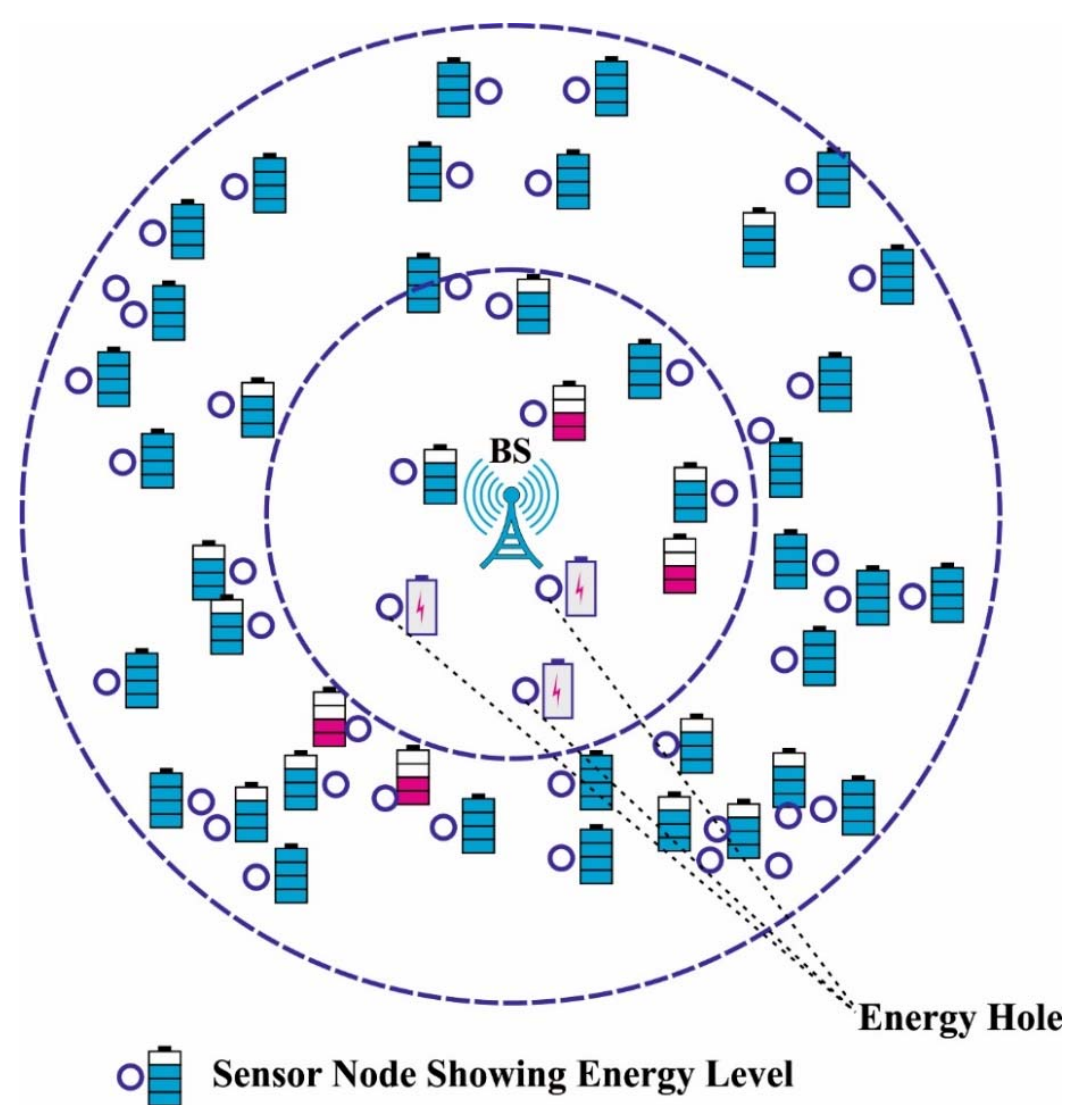

Fig. 1. Energy Hole problem in WSN

In WSN, clustering is one of the effective modules used for conserving the power and expanse the system lifespan [3]. According to the RE of node and average energy of a cluster, Adaptive Decentralized Re-clustering Protocol (ADRP) is used for selecting the collective cluster head $(\mathrm{CH})$ for consecutive iterations [4]. However, new nodes cannot be supplemented till reaching the forthcoming phase and even if a node is added then the node dies, and cluster formation would be terminated. In [5], developers reduce the count of $\mathrm{CH}$ broadcasting to best number of $\mathrm{CH}$ count in $\mathrm{CH}$ election process to preserve the energy. Numerous clustering protocols were projected in diverse studies; however, the energy hole problem is not considered.

This paper presents an efficient Mitigating Energy Hole based routing algorithm (MEHRA) for WSN. Initially, the network initialization process takes place where the entire network is partitioned into few regions and is again divided into sectors with respect to equiangular wedges. During the partitioning of the network as to regions and wedges, Head Node (HN) will be chosen for all sectors regarding distance as well as energy. Besides, the sensor nodes in a provided sector method a chain to communicate with their HN. The construction of chain process has begun from the farther node from the $\mathrm{HN}$ and each node is choosing its chain neighboring by the execution of greedy method. The HN collects the data from its predecessor and its individual sector and transmits the aggregated data to its successor HN till it reaches the BS. An extensive set of experimental analysis was carried out to highlight the supremacy of the MEHRA technique.

\section{Literature Survey}

[6] projected a cluster-relied protocol according to the virtual grid structure as well as mobile sink to apply the power utilization of sensor nodes by mitigating the traffic load. The nodes which are closer to the intersecting region of virtual grid are in charge of monitoring the current location of mobile sink. A sensing node forwards the data to mobile sink under the application of geographic routing approach that depends upon position details collected from intersecting regions. [7] deployed Multi-Hop Energy Efficient Routing Protocol using Multiple Mobile Nodes (MEACBM) protocol for WSN. A novel probabilistic CH election approach and develop numerous clusters as well as sub-clusters. Next, sensing region is classified as various sectors and a sector has numerous clusters as well as sub-clusters.

Multilayer Cluster Designing Algorithm (MCDA) [8] developed to enhance the power efficacy of Distributed Cluster Designing (DCD) as well as Centralized Cluster Designing scheme (CCD). The newly developed protocol is composed of 3 phases namely, self-organizing, flat layer design as well as clustered layer design. Therefore, nodal density is the basic measure used in $\mathrm{CH}$ election process repeatedly. Moreover, count of Cluster Member 
(CM) for a cluster is undefined. [9] developed energy hole repelling approach where the sensor nodes placed nearby the sink develops tiny clusters while nodes placed away sink BS makes huge clusters. [10] deployed an Energy Efficient Unequal Chain Length Clustering (EEUCLC) protocol to resolve the energy hole issues by making UC inside a cluster. [11] introduced UC model to eliminate the hot spot problem, by developing tiny cluster placed closer to the sink and huge cluster are located away from the sink node. It is also used for load distribution; however, it does not make sure the efficiency when the energy hole problem is aroused. [12] established a layer-based UC approach with the help of multi hop transmission to eliminate the issues.

[13] established Immune Clone Selection-based Power Control (ICSPC) scheme to resolve the energy hole problem with the help of cluster relied upon coronal method. [14] established On-hole children reconnection (OHCR) by concerning local nature and on-hole alert (OHA) by global nature protocols and connect to network even after the node expiry. It is due to the sudden death of parent node and the child nodes explore a new parent node with limited ranges. For chain based system, it makes alternate chain head. [15] deployed Prolong Stable Election Protocol (P-SEP) for expanding the network lifespan and to increase the power efficacy by the optimal $\mathrm{CH}$ election in a fog computation. Here, the fog nodes are useful to send the collected data to internet gateway which has to be placed in sensing area. A protocol adopts 2-level heterogeneity on the basis of energy and divides the sensor nodes into 2 classes namely, advanced as well as normal node. Mostly, the sensor nodes are deployed in remote areas and mitigate the development in real-time application because of the previous placement demand.

\section{Problem Formulation}

Assume a multihop WSN in which maximum count of sensor nodes are distributed in the monitoring region and forward the sensed data to BS. With no loss in generality, consider that the nodes are placed equally with a density of $\rho$. The monitoring region is classified as $N$ concentric blocks $\left\{C_{i}, i=1,2, \ldots, N\right\}$ placed at BS identical width $r$. The expressions are simplified and the sink node is represented as $C_{0}$. For simple analysis, data collection model is classified into multiple time slots. In all time slots, a node produces $l$ bits data and forwards a data rate $R$ to BS in a hop-by-hop manner. Also, nodes are duty-cycled to conserve the power which means that data transmission is carried out in active state. Once the transmission is completed, it returns to the sleep state till the consecutive time slot is initialized. Also, node wishes to send the data to relay node based on the linear route to BS with distance $r$.

In classical non-cooperative transmission procedure, all data has been forwarded by using single-input singleoutput (SISO_model. Nodes present in corona $C_{i}$ send $L_{i}$ bits data produced by themselves and $L_{i+1, i}$ bits data forwarded from $C_{i+1}$ is produced by $\left.\left\{C_{j} \mid i+1 \leq j \leq N\right\}\right)$ to $C_{i-1}$, where

$$
\begin{gathered}
L_{i+1, i}=\left(N^{2}-i^{2}\right) \pi r^{2} \rho l \\
L_{i}=\left[i^{2}-(i-1)^{2}\right] \pi r^{2} \rho l=(2 i-1) \pi r^{2} \rho l
\end{gathered}
$$

Nodes in $C_{i-1}$ gains the data and $L_{i, i-1}$ bits, and proceed with data transmission and expressed as,

$$
L_{i, i-1}=L_{i+1, i}+L_{i}=\left[N^{2}-(i-1)^{2}\right] \pi r^{2} \rho l
$$

It is pointed that nodes from $C_{N}$ has to send the corresponding data. In corona $C_{i}$, average data is loaded for every node, and $\bar{L}_{i-1, i}$, is represented by,

$$
\bar{L}_{i, i-1}=\frac{L_{i, i-1}}{(2 i-1) \pi r^{2} \rho}=\frac{\left[N^{2}-(i-1)^{2}\right]}{(2 i-1)} l
$$

It is apparent that nodes available in corona $C_{1}$ has enormous traffic load and results in robust power dispersion. When the battery power is drained from these nodes, the energy hole problem or hot spot issues have been aroused.

\section{The Proposed Method}

The entire network is classified into coronas in the network initialization. Then, the coronas are further categorized as sectors with respect to equiangular wedges. When the network is classified as corona and wedges, HN is selected for all sectors by concerning the distance as well as power. Sensor nodes in applied sector make a chain for communicating with $\mathrm{HN}$. The chain development is initialized from the distance node of $\mathrm{HN}$ and each node selects the chain neighbor using greedy approach. Moreover, the HN gathers data from predecessor and respective sector would forward accumulated data to the successor $\mathrm{HN}$ till it is received by sink node. 


\subsection{Classify the network as coronas}

In network established state, BS classifies a network as circularly structured coronas by changing the communication energy. Then, sink node changes the transmission energy as high communication radius and fix the corona value as $0(\mathrm{CN}=0)$. When the corona value is increased by, BS telecasts a corona creation message. The node that receives corona message with $\mathrm{CN}=\mathrm{I}$ fix the corona to $\mathrm{i}$, until it takes combined with symmetric or minimum corona. It is repeated till the nodes receive the corona value.

\subsection{Divide the network into wedges}

In order to classify the network as equiangular wedges, sink node directs the respective antenna to a part of network and forwards a beacon. Then, beacon is forwarded with higher communication energy level which is composed of a wedge identifying devise. Next, extended angle of directionality BS forwards as well as re-telecasts novel wedge identifier which is repeated still the BS announces wedge identifier beacon to whole system. Once the corona development is completed while wedge sensor node has gained the similarity of corona as well as wedge. The location of sensor nodes with identical coordinates forms a sector, in shape of hard disk. BS verifies the sector having maximum number of nodes. Sector size differs on the basis of DBS, sector nearby sink node is minimum and intra sector cost among the sector's node is limited and it has to apply minimal communication power.

\subsection{Data Communications}

It is comprised of 3 states namely HN election to a sector, Chain development, and Data transmission. The HN from a sector forwards the accumulative traffic emerged in the predecessors and respective sectors to the successors. Since a single node from a sector is applied for transmitting the data to sink; the medium access control (MAC) level intensity is minimum. The newly developed model deals with BS location which is assumed to be the middle portion of the system. Thus, $\mathrm{HN}$ election process is initialized from internal sector of sink node. Every sector would be comprised of single HN and the election is based on node's RE as well as distance with relative successor. A node allocates a timer (Ti) based on the given expression,

$$
T i=(\text { Einit } / E i)+(\text { DitoHN })
$$

In this model, Einit is referred to be the primary energy of a node, Ei indicates node's RE, DitoHN represents the distance among nodes and successor HN. Therefore, node with timer elapses to 0 and broadcast HN_Msg to the sector and update the alternate node to move away from the HN election competition. Sensor nodes from same sector would leave the competition. An HN announces respective position details to adjacent $\mathrm{HN}$ of a sector. In a sector, the chain development might be simulated from the distant node under the application of Greedy approach. Once the $\mathrm{HN}$ election is completed, high distance node from $\mathrm{HN}$ is elected and develops the node chain. A sensor node of a sector applies greedy model for creating a short distance node as the upcoming node makes cycle. Excluding HN, not all nodes could transmit or receive the data from alternate nodes. The sensor node acquires the time division multiple access (TDMA) schedule for data communication to corresponding HN. The nodes in every sector forward the data to closer node within a limited time period. The data transmission is initialized from external coronas. If $\mathrm{HN}$ is available inside corona 1, a sector gains the data from predecessor sector, after accumulation the data is forwarded to BS.

\subsection{Merging process of a wedge}

In $\mathrm{HN}$ identifies that the sector has limited average energy when compared with initial energy, which has to merge the sectors. Initially, wedge merging is performed and the sector with minimum average energy from adjacent $\mathrm{HN}$ announces the sector's average energy by transmitting a beacon called avg-en. Since the $\mathrm{HN}$ is aware of the power information, it relies on forwarding average energy data to HN. Once the reply has arrived from a neighbor HN, a sector having maximum power is decided for merging. Consider that sector in corona- 2 and wedge- 2 position refers $(2,2)$ position is comprised of minimum power and starts merging, which ask sector $(2,3)$ and $(2,1)$ to send the average energy. The sector $(2,3)$ would be chosen to merge as the sector contains maximum average energy when compared with the sector $(2,1)$. Initially, sectors $(1,3)$ and $(1,2)$ combines and the node from sector $(1,2)$ inform the sector as $(1,3)$. Afterward, sector $(2,2)$ and $(2,3)$ is merged and it is repeated still the external sector of lower energy wedge sector is merged. A node which has been merged with a sector updates the location on the basis of new wedge. In line with this, the sector having low as well as high energy wedge would be merged and considered as single wedge. The neighbor of unified wedge upgrades the neighbor data.

\section{Experimental Validation}

Fig. 2 depicts the alive node analysis of MEHRA method with previous MCDA, EEEHR, and CCS schemes under the presence of 100 nodes. The figure portrayed that the MEHRA has accomplished expanded network lifetime when compared with classical methods. Simultaneously, the CCS and MCDA methods have depicted least 
network lifetime while considerable network lifetime is exhibited by the EEEHR model. For sample, on the execution round of 1000, greater value of 30 nodes are alive by the MEHRA method and lower of 4, 4, and 22 nodes are alive by the CCS, MCDA, and EEEHR methods. Likewise, on the execution round of 2000, a maximum alive node of 15 has been provisioned by the newly developed MEHRA model while a limited number of 0,2 , and 12 nodes are alive by the presented model.

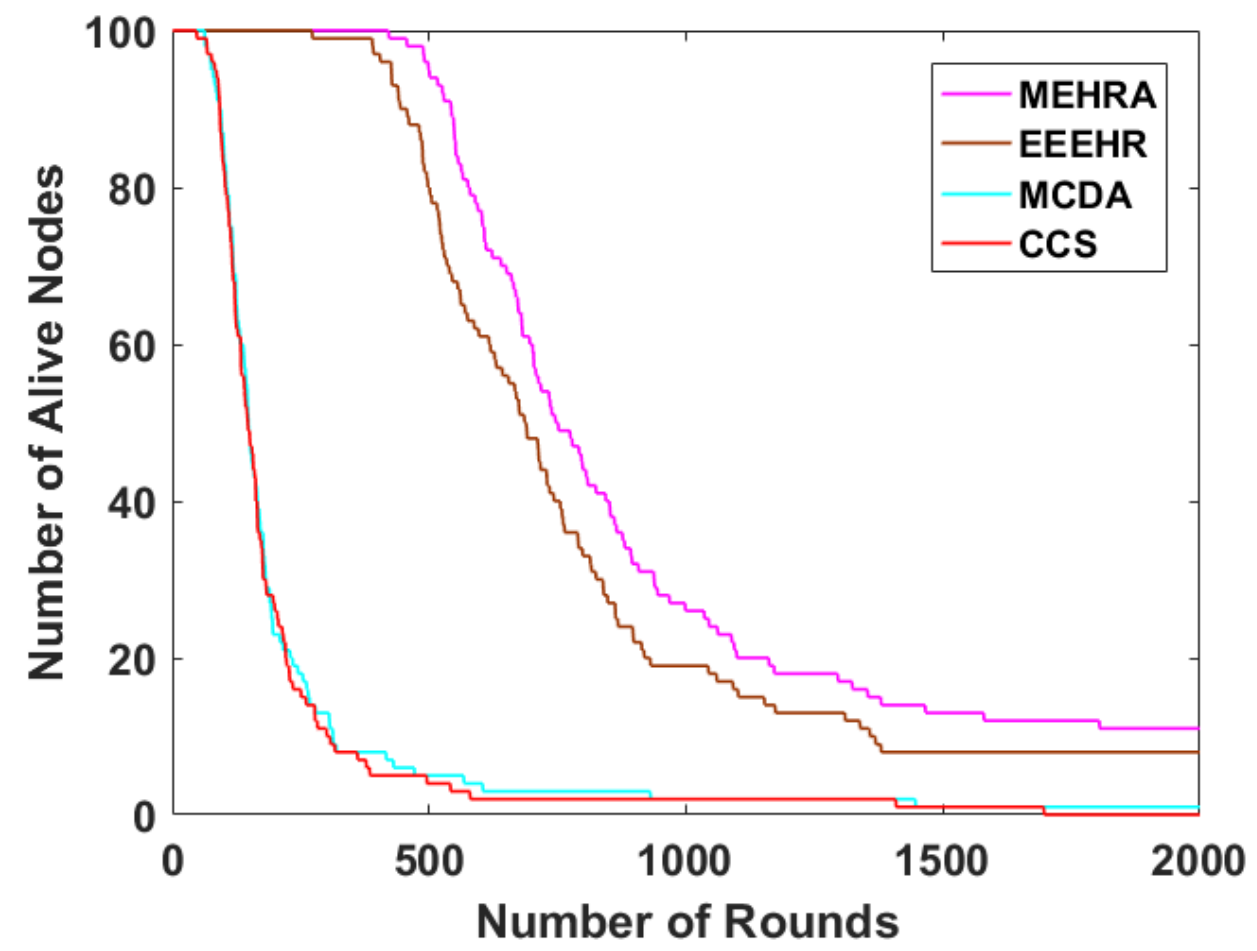

Fig. 2. Alive node analysis of MEHRA technique under 100 nodes

Fig. 3 demonstrates the active node analysis of MEHRA scheme with existing MCDA, EEEHR, and CCS techniques under the existence of 150 nodes. The figure portrayed that the MEHRA has achieved improved network lifetime than its comparatives. Concurrently, the CCS and MCDA models have showcased minimum network lifespan whereas slightly improved network duration was reached by the EEEHR framework. For instance, on the execution round of 1000 , a greater number of 54 nodes are active by the MEHRA model whereas minimum of 0,2 , and 30 nodes are alive by the CCS, MCDA, and EEEHR methods. In line with this, in the execution round of 2000, enormous alive node of 13 has been maintained by the newly developed MEHRA method and reduced number of 0,1 , and 9 nodes are alive by the proposed model. 


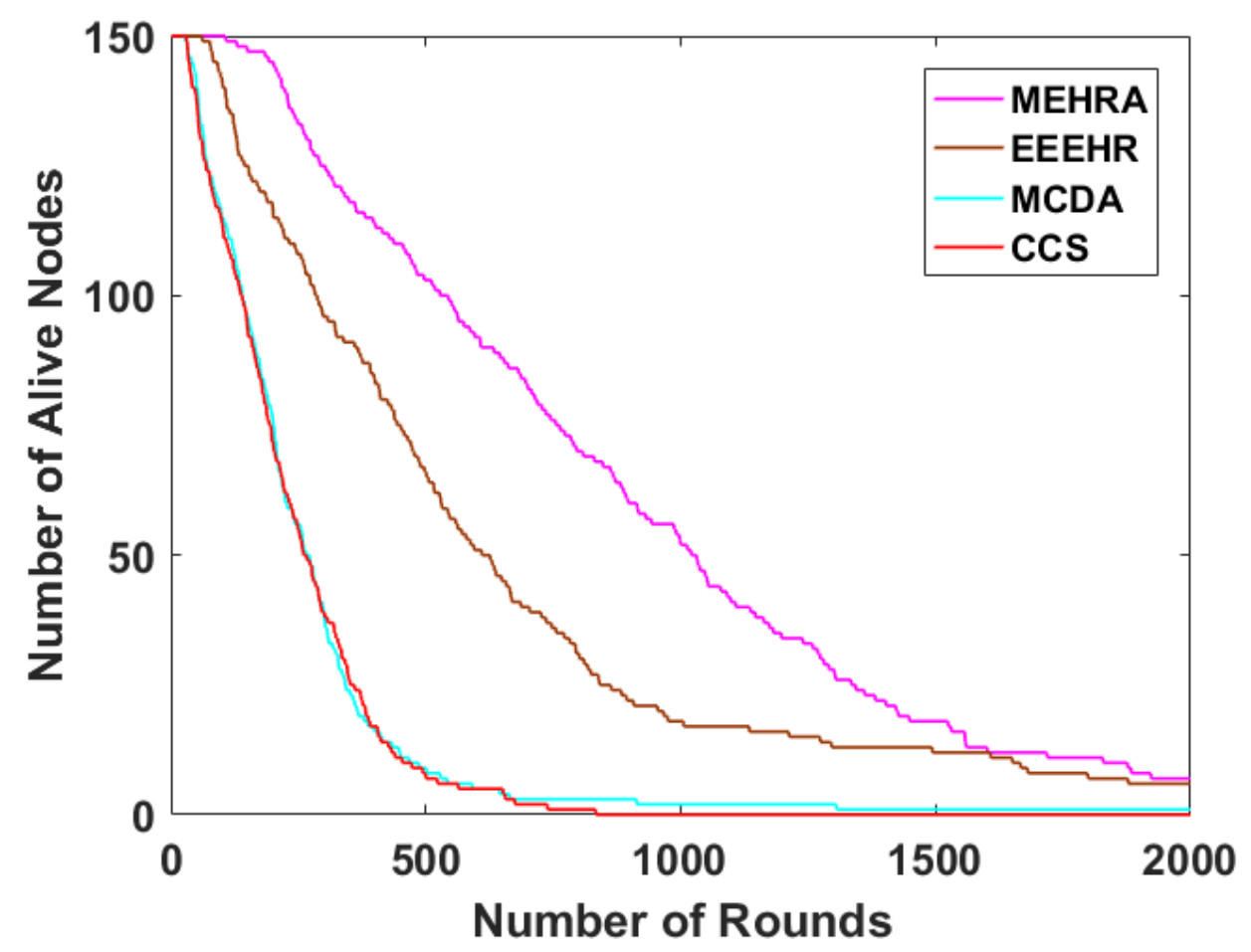

Fig. 3. Alive node analysis of MEHRA technique under 150 nodes

Fig. 4 illustrates the alive node analysis of MEHRA approach with traditional MCDA, EEEHR, and CCS methodologies under the existence of 250 nodes. The figure indicated that the MEHRA has reached improvised network duration when compared with the traditional approaches. Meantime, the CCS and MCDA methods have depicted minimum network duration whereas a reasonable network lifespan is accomplished by EEEHR scheme. For instance, on the execution round of 1000, a maximum number of 82 nodes are alive by the MEHRA model and the least count of 2, 8, and 70 nodes are alive by the CCS, MCDA, and EEEHR frameworks. Similarly, on the execution round of 2000, a qualified alive node of 20 has been retained by the presented MEHRA approach whereas a reduced number of 0,2 , and 18 nodes are alive by the projected scheme.

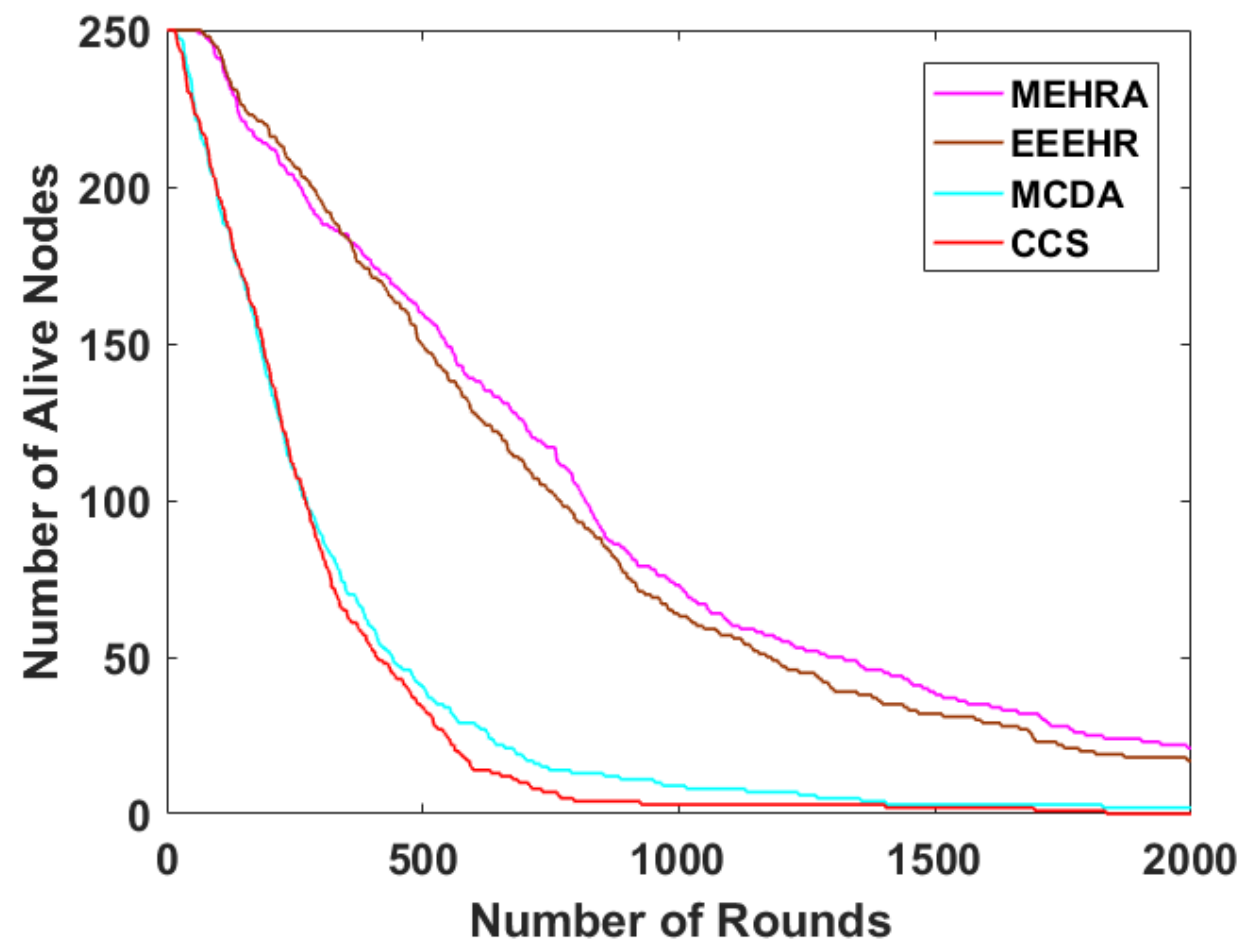

Fig. 4. Alive node analysis of MEHRA technique under 250 nodes 


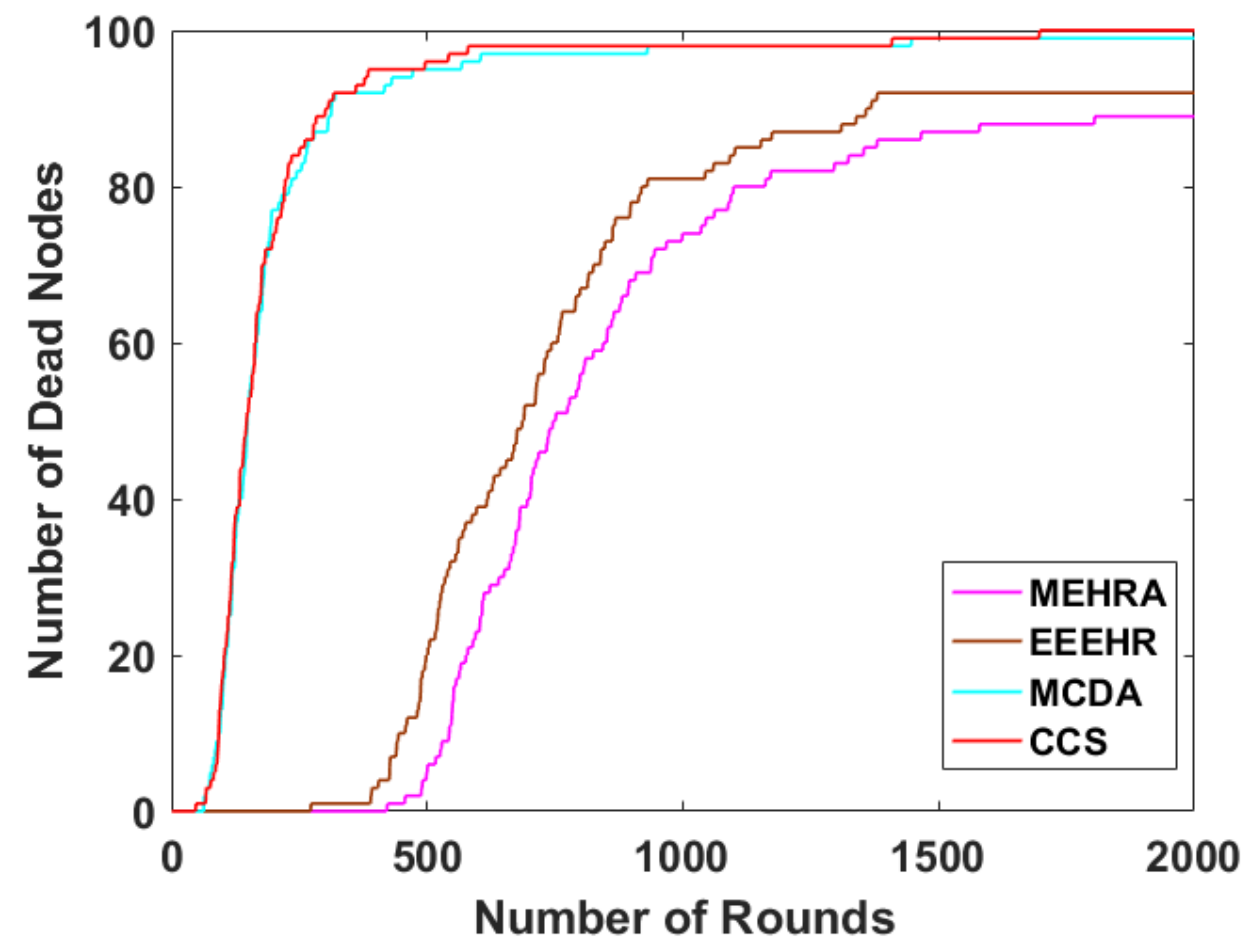

Fig. 5. Dead node analysis of MEHRA technique under 100 nodes

Fig. 5 verifies the dead node investigation of MEHRA technique with the accessible MCDA, EEEHR, and CCS methods under the application of 100 nodes. The figure demonstrated that the MEHRA has accomplished maximum network duration when compared with the earlier models. At the same time, the CCS and MCDA models have assured lower network lifespan while moderate network lifetime is exhibited by the EEEHR approach. For example, on the execution round of 1000, a low value of 70 nodes are dead by the MEHRA method while high value of 96, 96, and 78 nodes are dead by the CCS, MCDA, and EEEHR methodologies. Along with that, on the execution round of 2000, the minimum count of 85 nodes is expired by the newly developed MEHRA scheme and limited number of 100, 98, and 88 nodes are dead by the CCS, MCDA, and EEEHR models.

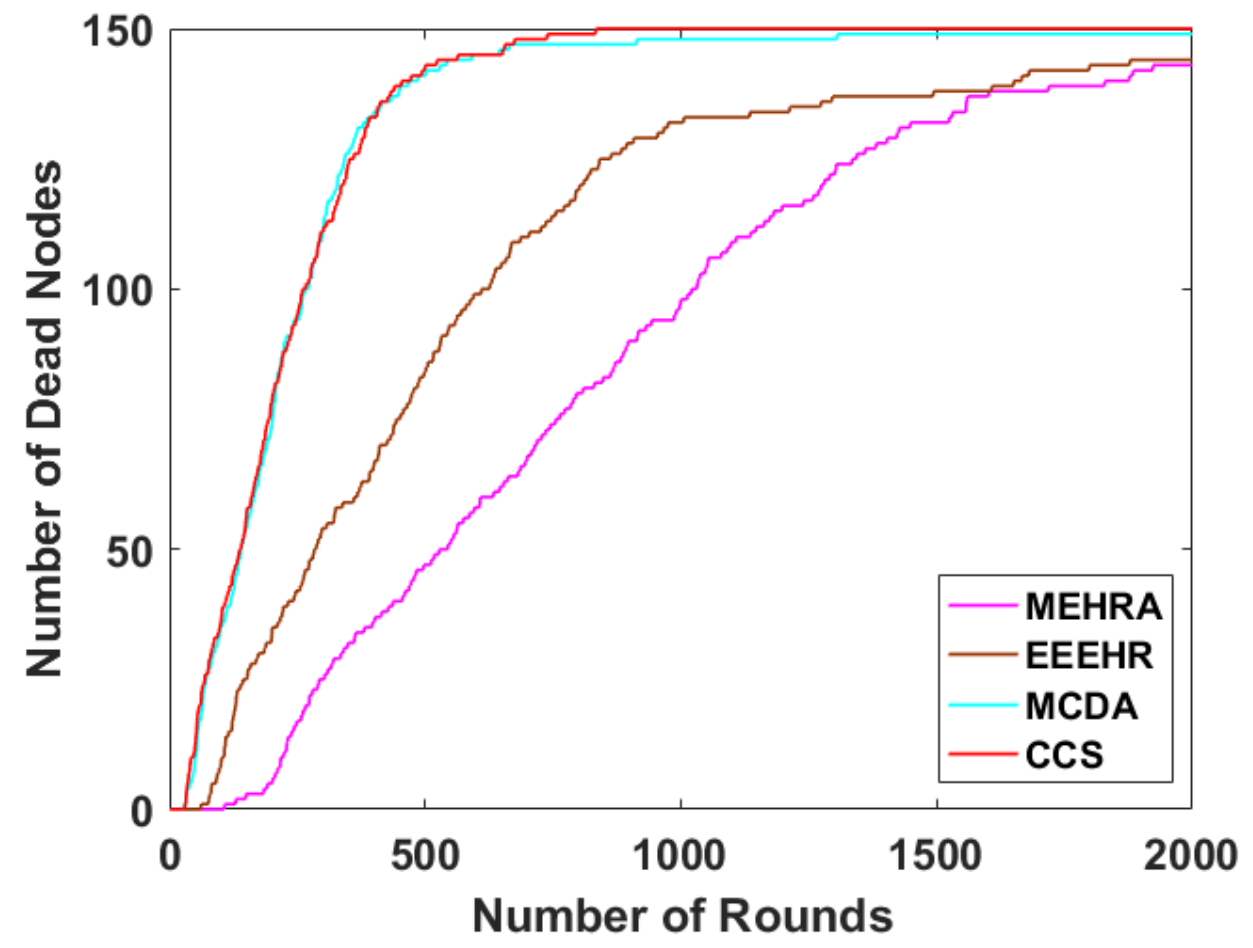

Fig. 6. Dead node analysis of MEHRA technique under 150 nodes 
Fig. 6 inspects the dead node analysis of MEHRA framework with the available MCDA, EEEHR, and CCS schemes under the presence of 150 nodes. The figure demonstrated that the MEHRA has achieved expanded network lifespan over the compared techniques. Meantime, the CCS and MCDA methodologies have confirmed minimum network lifespan whereas a considerable network lifespan has been accomplished by EEEHR method. For instance, on the execution round of 1000, a minimum of 96 nodes are dead by the MEHRA approach whereas high count of 150,148, and 120 nodes are expired by the CCS, MCDA, and EEEHR frameworks. Similarly, in the execution round of 2000, lower count of 137 nodes are inactive by the presented MEHRA approach whereas least count of 150, 149, and 141 nodes are expired by the CCS, MCDA, and EEEHR methods.

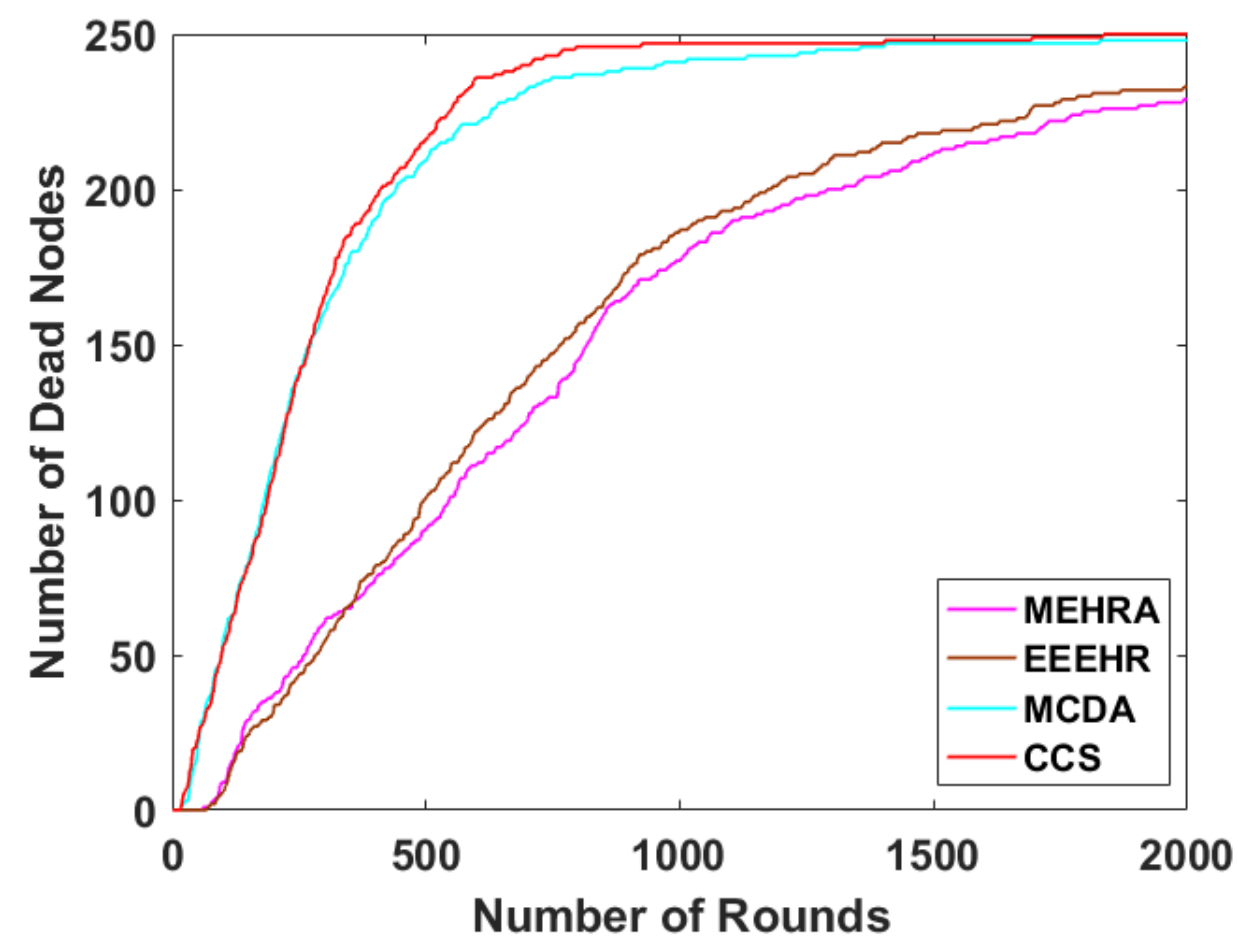

Fig. 7. Dead node analysis of MEHRA technique under 250 nodes

Fig. 7 validates the dead node investigation of MEHRA approach with the available MCDA, EEEHR, and CCS techniques under the application of 250 nodes. The figure portrayed that the MEHRA has achieved improved network duration than its comparatives. At the same time, the CCS and MCDA models have confirmed minimum network duration whereas acceptable network lifespan has been obtained by the EEEHR techniques. For instance, on the execution round of 1000, lower 168 nodes are inactive by the MEHRA method and maximum count of 248, 242, and 170 nodes are expired by the CCS, MCDA, and EEEHR methodologies. In line with this, on the execution round of 2000, a minimum number of 230 nodes are expired by the newly developed MEHRA method and minimum count of 250, 248, and 232 nodes are inactive by the CCS, MCDA, and EEEHR methods.

Fig. 8 verifies the EC investigation of the MEHRA method with the traditional methods under the existence of 50 nodes. The figure portrayed that the CCS model requires greater EC when compared with the classical schemes. Additionally, the EEEHR technique has applied moderate EC than CCS model and even lesser EC has been accomplished by the EEEHR model. Consequently, the MEHRA method has required the least EC under diverse rounds of operation. For example, under the execution round of 1000, the presented MEHRA scheme has required a higher EC of $15 \mathrm{~J}$ and the alternate models like CCS, MCDA, and EEEHR models require minimum EC of $2 \mathrm{~J}$, $2 \mathrm{~J}$, and $12 \mathrm{~J}$ correspondingly. Ultimately, under the operational round of 2000, the projected MEHRA model has reserved a considerable EC of $6 \mathrm{~J}$ while the CCS, MCDA, and EEEHR models require minimum EC of $4 \mathrm{~J}, 1 \mathrm{~J}$, and $0 \mathrm{~J}$ correspondingly. 


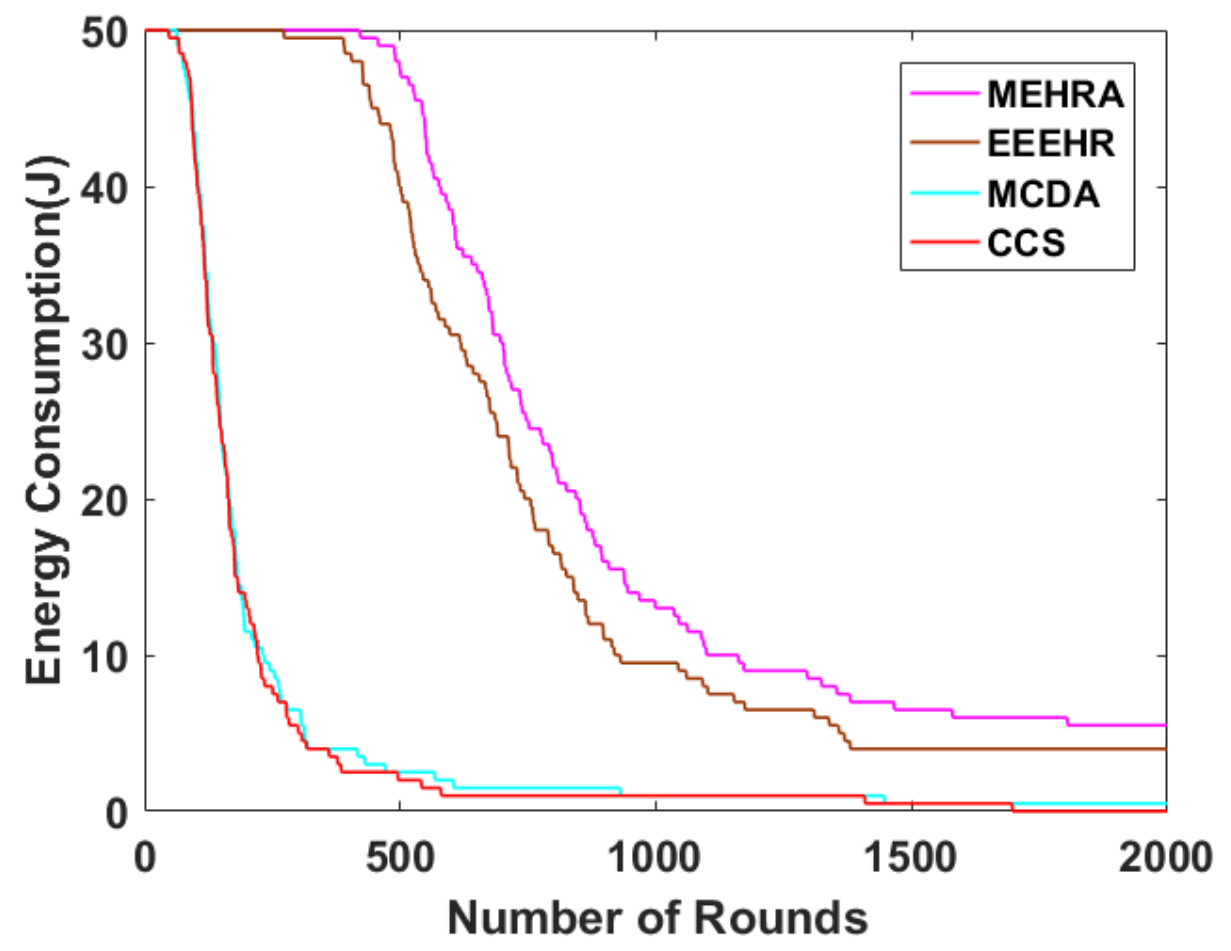

Fig. 8. EC analysis of MEHRA technique under 50 nodes

Fig. 9 reviews the EC examination of the MEHRA scheme with the former techniques under the presence of 80 nodes. The figure represented that the CCS technique has attained higher EC when compared with other models. Moreover, the EEEHR model has utilized considerable EC than CCS approach whereas even better EC has been attained by the EEEHR scheme. Consequently, the MEHRA approach has required a minimum EC under varying rounds. For example, under the execution round of 1000, the presented MEHRA model has required the minimum EC of 30J while the compared schemes namely, CCS, MCDA, and EEEHR models have acquired maximum EC of $0 \mathrm{~J}, 2 \mathrm{~J}$, and $17 \mathrm{~J}$ respectively. Besides, under the operational round of 2000, the presented MEHRA model has reserved an adaptable EC of $8 \mathrm{~J}$ whereas the CCS, MCDA, and EEEHR models require minimum EC of 5J, $1 \mathrm{~J}$, and $0 \mathrm{~J}$ correspondingly.

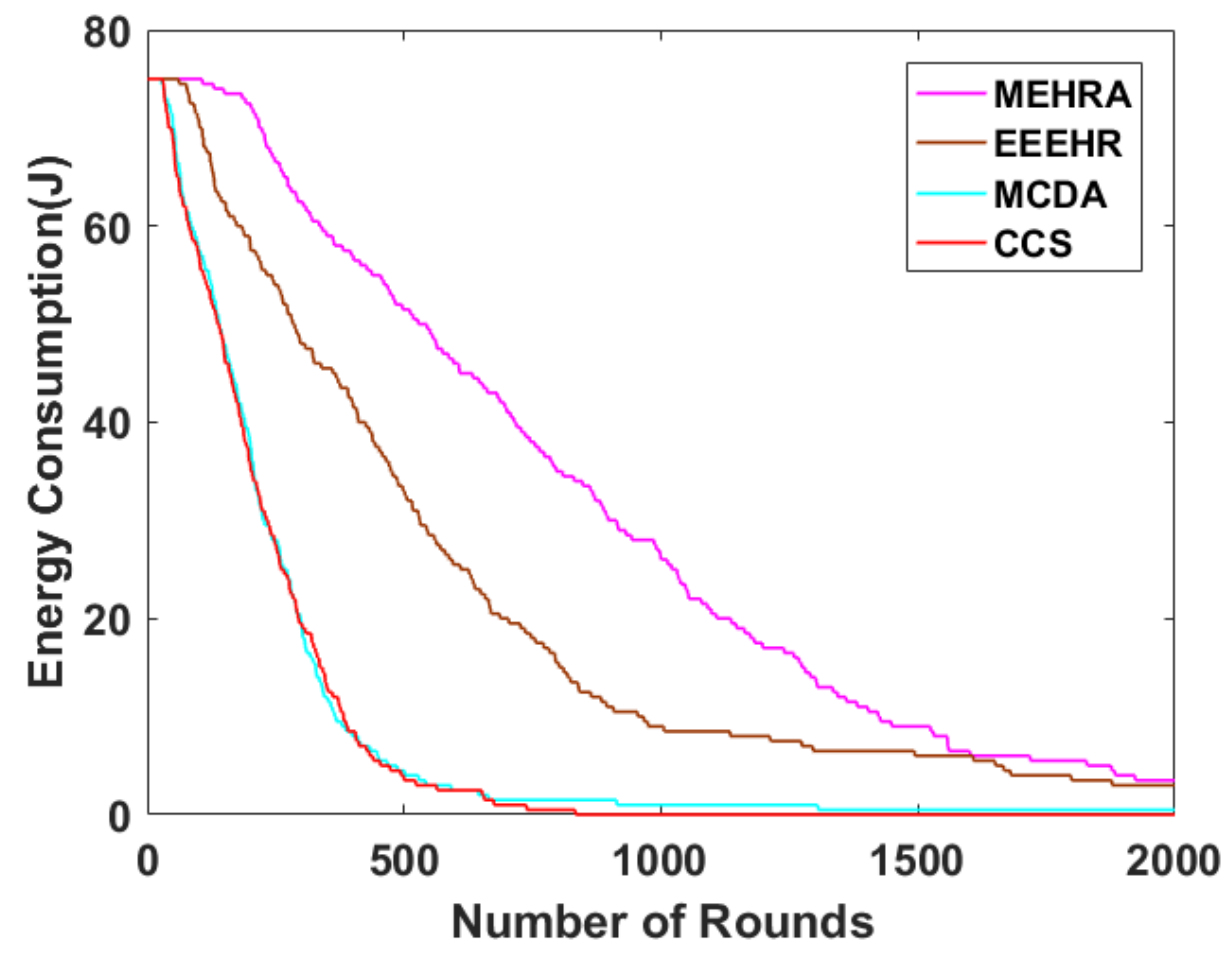

Fig. 9. EC analysis of MEHRA technique under 80 nodes 
Fig. 10 investigates the EC analysis of the MEHRA model with the conventional techniques under the presence of 140 nodes. The figure demonstrated that the CCS method has acquired maximum EC over the previous models. In addition, the EEEHR model has employed moderate EC over CCS scheme whereas acceptable EC has been accomplished by the EEEHR approach. At last, the MEHRA technique has necessitated least count of EC under varying rounds of operation. For instance, under the execution round of 1000, the presented MEHRA model has necessitated a minimum EC of 42J while the other models like CCS, MCDA, and EEEHR models have required greater EC of $3 \mathrm{~J}, 9 \mathrm{~J}$, and $38 \mathrm{~J}$ correspondingly. Next, under the operational round of 2000, the newly developed MEHRA model has booked a considerable EC of 16J and CCS, MCDA, and EEEHR models requires minimum EC of $0 \mathrm{~J}, 2 \mathrm{~J}$, and $14 \mathrm{~J}$ correspondingly.

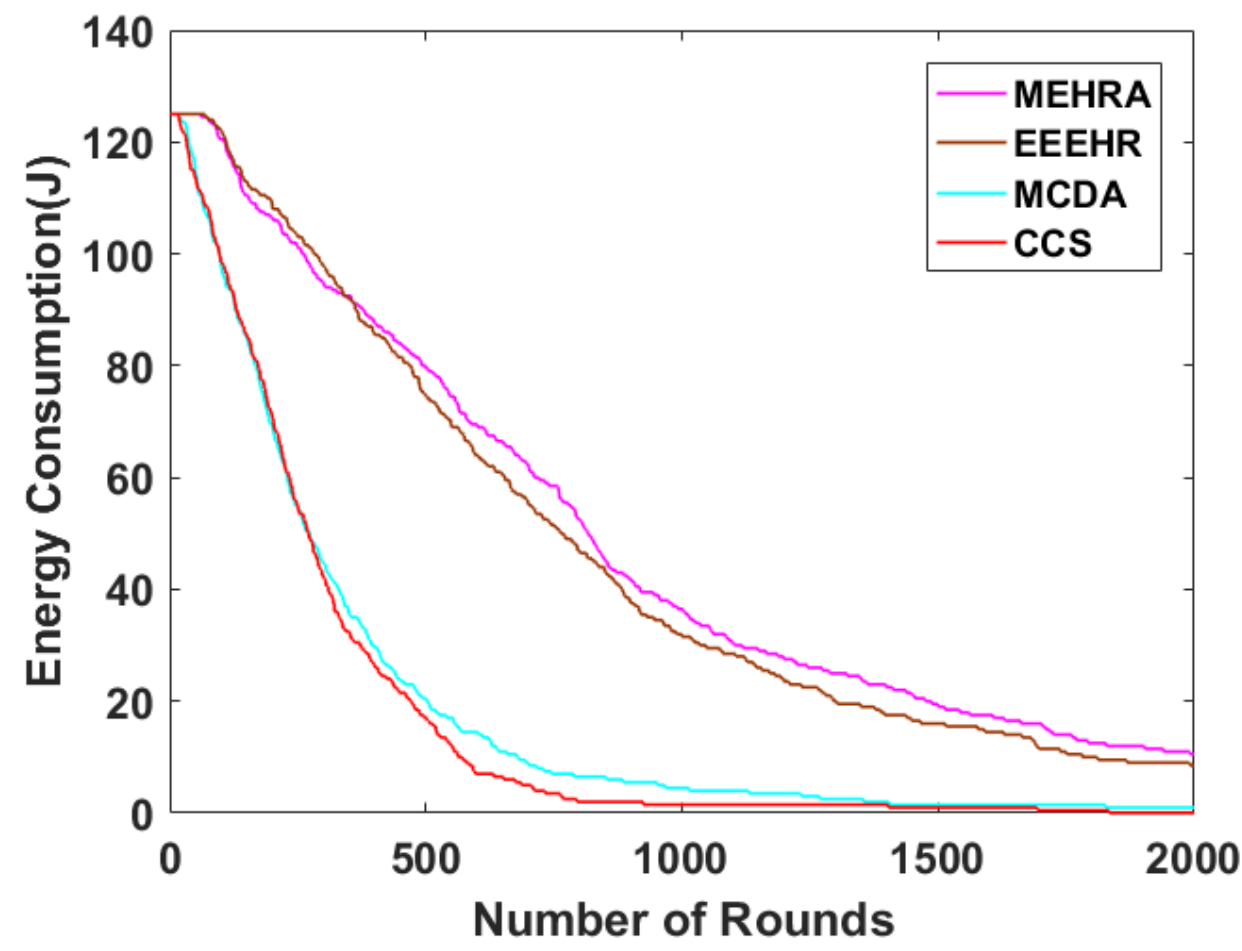

Fig. 10. EC analysis of MEHRA technique under 140 nodes

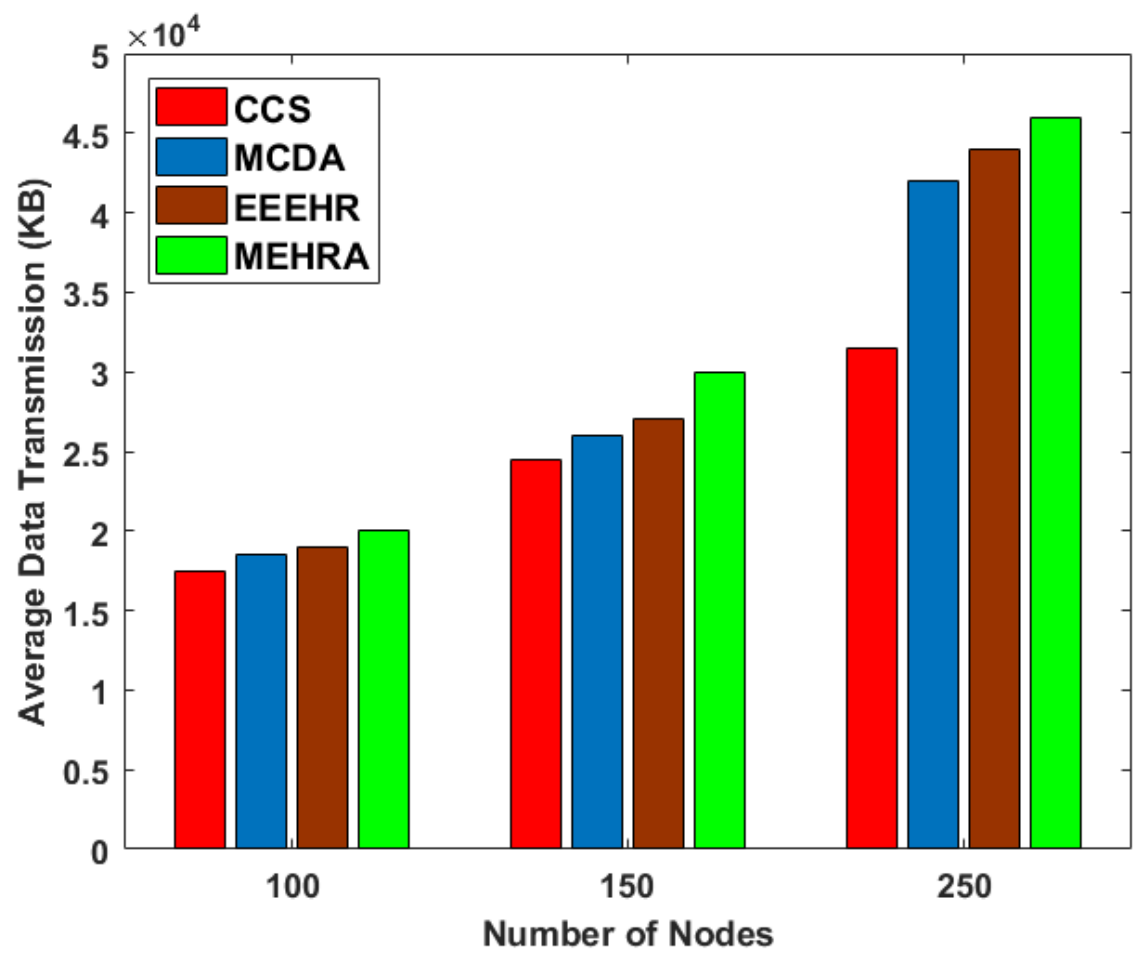

Fig. 11. Average Data Transmission Analysis of MEHRA Technique 
Fig. 11 examines the average data transmission investigation of the proposed MEHRA model under diverse percentage of clusters. The figure portrayed that the CCS approach has failed to display considerable performance by achieving limited count of average data transmission. Meantime, the MCDA and EEEHR methods have displayed moderate and similar results by reaching acceptable count of average data transmission. Finally, the MEHRA approach has provided an improved performance with the supreme average data transmission.

\section{Conclusion}

This paper has presented a novel MEHRA protocol to resolve energy hole problem in WSN. In MEHRA technique, the entire network is classified into coronas in the network initialization. Then, the coronas are further categorized as sectors with respect to equiangular wedges. When the network is classified as corona and wedges, the $\mathrm{HN}$ is selected for all sectors by concerning the distance as well as power. Sensor nodes in applied sector make a chain for communicating with HN. The chain development is initialized from the distance node of HN and each node selects the chain neighbor using greedy approach. Moreover, the HN gathers data from predecessor and respective sector would forward accumulated data to the successor HN till it is received by sink node. A wide range of simulations was performed to ensure the goodness of the presented technique and the resultant values verified the goodness of the MEHRA technique interms of distinct aspects.

\section{References}

[1] Akyildiz, I.F.; Su, W.; Sankarasubramaniam, Y.; Cayirci, E. Wireless sensor networks: A survey. Comput. Netw. 2002, 38, $393-422$.

[2] Naureen, A.; Zhang, N.; Furber, S. Identifying Energy Holes in Randomly Deployed Hierarchical Wireless Sensor Networks. IEEE Access 2017, 5, 21395-21418.

[3] Lu, H.; Li, J.; Wang, G. A novel energy efficient routing algorithm for hierarchically clustered wireless sensor networks. In Proceedings of the Fourth International Conference on Frontier of Computer Science and Technology, Shanghai, China, 17-19 December 2009; pp. $565-570$.

[4] Bajaber, F.; Awan, I. Adaptive decentralized re-clustering protocol for wireless sensor networks. J. Comput. Syst. Sci. 2011, 77, 282292.

[5] Batra, P.K.; Kant, K. LEACH-MAC: A new cluster head selection algorithm for Wireless Sensor Networks. Wirel. Netw. 2016,22 , 49.

[6] Yarinezhad, R.; Sarabi, A. Reducing delay and energy consumption in wireless sensor networks by making virtual grid infrastructure and using mobile sink. AEU-Int. J. Electron. Commun. 2018, 84, 144-152.

[7] Toor, A.S.; Jain, A. Energy Aware Cluster Based Multi-hop Energy Efficient Routing Protocol using Multiple Mobile Nodes (MEACBM) in Wireless Sensor Networks. AEU-Int. J. Electron. Commun. 2019, 102, 41-53.

[8] Jabbar, S.; Minhas, A.A.; Paul, A.; Rho, S. Multilayer cluster designing algorithm for lifetime improvement of wireless sensor networks. J. Supercomput. 2014, 70, 104-132.

[9] Prabha, K.L.; Selvan, S. Energy Efficient Energy Hole Repelling (EEEHR) Algorithm for Delay Tolerant Wireless Sensor Network. Wirel. Pers. Commun. 2018, 101, 1395-1409.

[10] Baniata, M.; Heo, M.; Lee, J.; Park, J.W.; Hong, J. Energy-efficient unequal chain length clustering for WSN. In Proceedings of the 33rd Annual ACM Symposium on Applied Computing, Pau, France, 9-13 April 2018; pp. 2125-2131.

[11] Elkamel, R.; Messouadi, A.; Cherif, A. Extending the lifetime of wireless sensor networks through mitigating the hot spot problem. J. Parallel Distrib. Comput. 2019, 133, 159-169.

[12] Wang, Z.; Qin, X.; Liu, B. An energy-efficient clustering routing algorithm for WSN-assisted IoT. In Proceedings of the 2018 IEEE Wireless Communications and Networking Conference (WCNC), Barcelona, Spain, 15-18 April 2018; pp. 1-6.

[13] Zhao, X.; Xiong, X.; Sun, Z.; Zhang, X.; Sun, Z. An immune clone selection based power control strategy for alleviating energy hole problems in wireless sensor networks. J. Ambient. Intell. Humaniz. Comput. 2019, 1-14.

[14] Mohemed, R.E.; Saleh, A.I.; Abdelrazzak, M.; Samra, A.S. Energy-efficient routing protocols for solving energy hole problem in wireless sensor networks. Comput. Netw. 2017, 114, 51-66.

[15] Naranjo, P.G.V.; Shojafar, M.; Mostafaei, H.; Pooranian, Z.; Baccarelli, E. P-SEP: A prolong stable election routing algorithm for energy-limited heterogeneous fog-supported wireless sensor networks. J. Supercomput. 2017, 73, 733-755. 